\title{
Hypoxia-Related Gene FUT11 Promoted Pancreatic Cancer Progression Via Maintaining The Stabilization of PDK1
}

\section{Wenpeng Cao}

Guizhou Medical University

\section{Zhirui Zeng}

Guizhou Medical University

\section{Zhiwei He}

The Affiliated Hospital of Guizhou Medical University

\section{Runsang Pan}

Guiyang Maternity and Child Health Hospital

\section{Hao Wu}

The Affiliated Hospital of Guizhou Medical University

\section{Xiangyan Zhang}

Guizhou Provincial People's Hospital

\section{Hui Chen}

Guizhou Medical University

\section{Yingjie Nie}

Guizhou Provincial People's Hospital

Shan Lei ( $D 1109974497 @ q q . c o m)$

Guizhou Medical University

\section{Zijiang Yu}

Guizhou Medical University

\section{Research}

Keywords: pancreatic cancer, hypoxia, fucosyltransferase 11, hypoxia-inducible factor 1a, pyruvate dehydrogenase kinase 1

Posted Date: January 27th, 2021

DOl: https://doi.org/10.21203/rs.3.rs-152464/v1

License: (c) (1) This work is licensed under a Creative Commons Attribution 4.0 International License. Read Full License 


\section{Abstract}

Background: Hypoxia participated in the occurrence and development of pancreatic cancer (PC). However, genes associated with hypoxia respond and their regulated mechanism in PC cells were unclear. The current research was aimed to illuminate the role and hypoxia regulated mechanism of fucosyltransferase 11 (FUT11) in the progression of PC.

Methods: After predicting FUT11 as a key hypoxia associated gene in PC using bioinformatics analysis. The expression of FUT11 in PC using quantitative real-time fluorescent PCR, western blot and immunohistochemistry. The effects of FUT11 on PC cells proliferation, migration and invasion under normoxia and hypoxia were detected using Cell Counting Kit 8, 5-ethynyl-2'-deoxyuridine assay, colony formation assay and transwell assay. Spleen capsule injected liver metastasis and subcutaneously injected model were performed to confirm the effects of FUT11 in vivo. Furthermore, western blot, luciferase assay and immunoprecipitation were performed to explore the regulated relationship among FUT11, hypoxia-inducible factor 1a (HIF1a) and pyruvate dehydrogenase kinase 1 (PDK1) in PC.

Results: FUT11 was markedly increased of PC cells in hypoxia, up-regulated in the PC clinical tissues, and predicted a poor outcome. Inhibition of FUT11 reduced PC cell growth and mobility of PC cells under normoxia and hypoxia conditions in vitro, and growth and mobility in vivo. FUT11 bind with PDK1 and regulated the expression PDK1 under normoxia and hypoxia. FUT11 knockdown significantly increased the degradation rate of PDK1 under hypoxia, while treatment with MG132 can relieve the degradation of PDK1 induced by FUT11 knockdown. Overexpression of PDK1 in PC cells under hypoxia conditions reversed the suppressiv impacts of FUT11 knockdown on PC cell growth and mobility. In addition, HIF1a bound to the enhancer of FUT11 and increased its expression, as well as co-expressing with FUT11 in PC tissues. Furthermore, overexpress of FUT11 partially rescued the suppressiv effects of HIF1a knockdown on PC cell growth and mobility in hypoxia conditions.

Conclusion: Our data further implicate that hypoxia-induced FUT11 in PC contributes to proliferation and metastasis by maintaining the stability of PDK1, and suggest FUT11 maybe a novel and effective target for treatment of pancreatic cancer.

\section{Background}

Pancreatic cancer (PC) has high morbidity and mortality rates worldwide[1]. Even though the therapy method for PC such as operation, target therapy and chemotherapy had been developing, the number of tumor related mortality still increases every year[2]. Therefore, uncovering the molecular mechanism involved in PC and exploring the potential biomarkers for diagnosis and novel targets for treatment have recently emerged as a critical field.

The tumor microenvironment plays a vital role in the development of tumors and is closely related to the efficacy of tumor treatment. Targeting adaptive response for tumor environment may be a therapeutic strategy for PC[3]. Hypoxic microenvironment induces the up-regulation of a series of hypoxic-responsive 
genes, and then induce the proliferation, migration, drug resistance and various other biological events in cancer cells[4]. Hypoxia inducible factor 1-alpha (HIF1a) is a main regulator of cancer cells' transcriptional response to hypoxia, and up-regulates a series of genes that support tumor cells to compensate for the hypoxia microenvironment[5]. HIF1a overexpression has been detected in solid tumors and is related to the progression of a variety of cancers, including ovarian cancer[6], breast cancer[7], oropharyngeal squamous cell carcinoma[8], non-small cell lung cancer[9] and pancreatic cancer[10]. Studies have shown that HIF1a affects the regulation of tumor cell proliferation, angiogenesis, apoptosis and chemotherapy resistance during tumor development[11, 12]. However, the target genes of HIF1a in PC remain to be elucidated.

Fucosyltransferases (FUTs) were a family of enzymes which catalyzed the transfer process of fucose from GDP-fucose to glycoconjugates[13]. Various studies have demonstrated that FUT family is closely related to the occurrence and development of tumors. For example, Liang et a/ demonstrated that miR125a-3p/FUT5-FUT6 axis mediates the proliferation, mobility and pathological angiogenesis of colorectal cancer cells through the PI3K-Akt pathway [14]. Kumar et al exhibited that NCOA3 stabilized mucins post translationally through FUT8, which promoted the proliferation and metastasis of pancreatic cancer[15]. Lin et a/ reported that FUT11 and FUT1 genes were down-regulated in cisplatin-resistant variants cells [16]. However, the FUT11 functions and regulatory mechanisms in PC as yet remain largely unclear.

In our current study, we observed the FUT11 was a direct target gene of HIF1a, and it was up-regulated in PC cells under hypoxia, which can promote the proliferation and metastasis of PC cells via maintaining the stability of transcription factor 1 (PDK1) under hypoxia. Therefore, FUT11 may be a therapy target by blocking the hypoxia environment in PC.

\section{Materials And Methods}

\section{Bioinformatics analysis}

We downloaded Gene expression profile GSE67549 and GSE9350 from Gene Expression Omnibus database (GEO, https://www.ncbi.nlm.nih.gov/gds). GSE67549 contained 9 normoxic PC cells samples and 9 hypoxic PC cells samples, while GSE9350 contained 2 normoxic PC cells samples and 2 hypoxic $P C$ cells samples. Different expression genes were identified using the cut-off as Log2 fold change (FC) $>1$ and $P$ value $<0.05$. Common differently expression genes were analyzed using intersection analysis. The expression of these genes in the PC samples of The Cancer Genome Atlas (TCGA) and The Genotype-Tissue Expression (GTEx) database was determined using GEPIA (http://gepia.cancer-pku.cn/), $P<0.05$ was a threshold to consider significant.

\section{Clinical Samples}

Total 90 paired PC tissues and adjacent pancreatic tissues were provided by the PC patients which obtained surgery in the Affiliated Hospital of Guizhou Medical University. None of them obtained radiotherapy or chemotherapy prior to the surgery. The current study was approved by the Ethics 
Committee of Guizhou Medical University in accordance with Declaration of Helsinki, and patients who participated in the current study all obtained and signed the received informed consent.

\section{Cell culture and transfection}

Two human PC cell lines (PANC-1 and AsPC-1) used in the current study were obtained from ATCC. PANC1 and AsPC- 1 cells were incubated in DMEM with $10 \% \mathrm{FBS}$ at the $37^{\circ} \mathrm{C}$ environment with $5 \% \mathrm{CO} 2$. The condition of normoxia was set to $21 \% \mathrm{O}_{2}$ and $5 \% \mathrm{CO}_{2}$, while the condition of hypoxia was set to $1 \% \mathrm{O}_{2}$, $94 \% \mathrm{~N}_{2}$ and $5 \% \mathrm{CO}_{2}$. The FUT11 interference lentivirus and blank lentivirus plasmids were obtained from Sangon Biotech (Shanghai, China). PDX1 overexpression lentivirus was purchased from GeneChem (Shanghai, China). The small interfering RNAs (siRNAs) targeting HIF1a were obtained from JIMA (Shanghai, China). To construct the stable cell lines with target gene overexpression or knockdown, 1 $\mu \mathrm{g} / \mathrm{mL}$ puromycin (Sigma, USA) was added to continuously screen after transfection with lentivirus at $48 \mathrm{~h}$ for 10 days.

\section{Quantitative real-time fluorescent PCR}

Total RNA in PC tissues and cells was separated using TRIZOL (Beyotime Biotechnology, Hangzhou, China) and diluted into DNase/RNase-free water. After quantify, total RNA ( $2 \mu \mathrm{g}$ per sample) was reversely transcribed into cDNA using RevertAid First Strand cDNA Synthesis Kit (Fermentas, USA). Finally, qPCR was conducted to determine the expressed level of target genes using SYBR ${ }^{\text {TM }}$ Green PCR Master Mix (Solarbio, Wuhan, China). $\beta$-actin was set to the control. The primer sequences in our study were purchased from Tianyi Huiyuan (Wuhan, China) and shown as Table 1.

\section{Cell counting kit-8 (CCK-8) assay}

PANC-1 and AsPC-1 cells were plated in a 96-well plate in sextuplicate with $3 \times 10^{3}$ cells/well. Brief, $100 \mu \mathrm{l}$ DMEM medium contain 10 1 l CCK-8 regent (Boster, Wuhan, China) was added to each well in $24 \mathrm{~h}, 48 \mathrm{~h}$, $72 \mathrm{~h}$ and $96 \mathrm{~h}$ respectively. The absorbance of each well was detected at $450 \mathrm{~nm}$.

\section{5-ethynyl-2'-deoxyuridine (EDU) assay}

The EDU assay was carried out using a BeyoClick ${ }^{\mathrm{TM}}$ EdU-488 Proliferation Detection Kit (Beyotime, Suzhou, China). In brief, while PC cells adherence in 6-well plate, primary medium was removed and fresh medium were added. Then, total $10 \mu \mathrm{M}$ EDU was injected into each well and cells were cultured in $37^{\circ} \mathrm{C}$ for 2.5h. Following by fixation using $4 \%$ paraformaldehyde (Beyotime, Suzhou, China) for $15 \mathrm{~min}$ and

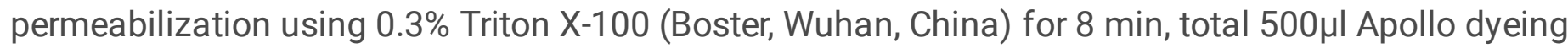
reaction buffer was then added for 40 min in dark environment. After staining the nuclear using DAPI for 10 min, the proportion of EDU was detected using fluorescence microscopy.

\section{Colony formation assay}


Cell with a density of 2000 cells/well were seeded into 6-well plates and cultured in DMEM media containing $10 \%$ FBS. After $24 \mathrm{~h}$, intervention factor was added and cultured for 2 weeks. After fixation in $4 \%$ paraformaldehyde for $15 \mathrm{~min}, 1 \%$ crystal violet was used to stain cell colonies. Cell colonies was counted and photographed.

\section{Western blotting}

The proteins in PC cells and tissues were extracted using RIPA reagent contain 5\% PMSF protease inhibitor. The BCA method was performed to detect the protein concentration of each sample. Proteins $(30 \mu \mathrm{g} / \mathrm{per}$ line) were added and separated by $10 \%$ SDS-PAGE for $120 \mathrm{~min}$. Then, the proteins were transferred into the PVDF membranes (Millipore, USA) with $0.45 \mu \mathrm{m}$ bore diameter, which was then seted in the environment contained $5 \%$ BSA for $30 \mathrm{~min}$ and incubated with primary antibody including FUT11, Ncadherin, E-cadherin, PDK1, HIF1 a and $\beta$-actin (all purchased from CST company) for $12 \mathrm{~h}$ in $4^{\circ} \mathrm{C}$. High sensitivity ECL reagent was used to visualize the blots in Multilmager and the relative expression of protein was calculated using Image J. $\beta$-actin was set as reference for FUT11, N-cadherin, E-cadherin, PDK1 and HIF1a.

\section{Transwell assay}

For transwell migration assay, total $4^{\prime} 10^{5}$ cells were suspended using $200 \mu \mathrm{l}$ DMEM medium without FBS

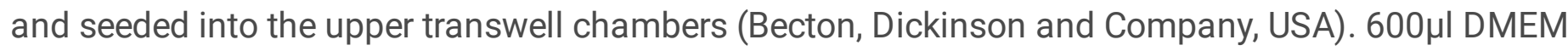
medium contained $10 \%$ FBS was placed in the lower transwell chambers. After $28 \mathrm{~h}$, migratory cells were fixed with paraformaldehyde and stained using $0.5 \%$ crystal violet. The transwell invasion experiment required matrigel(Becton, Dickinson and Company, USA) to be coated in the upper chamber before cell seeding. After fixation using $4 \%$ paraformaldehyde, staining with $1 \%$ crystal violet (Boster, Wuhan, China) and removing non-invasion cells using cotton swab. Finally, chambers were was photoed and counted.

\section{In vivo assay}

For subcutaneously injected model, total 10 female BALB/c nude mice were obtained from the animal central of Guizhou Medical University (Guizhou, China). After adaptive feeding, total $1 \times 10^{7}$ PANC- 1 cells with FUT11 knockdown and negative control cells were subcutaneously injected into upper-right flank of $B A L B / c$ mice( $n=5$ in each group). The health status of mice was monitored per day, while the tumor volume was detected per week. The tumor volume was monitored once a week and determined as followed: $\left(\mathrm{mm}^{3}\right)=\left(\right.$ Long $\left.\times W i d t h^{2}\right) / 2$. After 5 weeks. The protein level of KI67 and PCNA in tumor tissues was determined using immunohistochemical staining. For the metastasis assays, the metastasis ability of FUT11 knockdown and negative control group PANC-1 cell using spleen capsule injected liver metastasis model. FUT11 knockdown and negative control group PANC-1 cells ( $1^{\prime} 10^{7}$ cells ) were injected into the spleen of BALB/c mice ( $n=5$ in each group). Mice were sacrificed 12 weeks after inoculation, the liver tissues were harvested and photoed. HE stain was used to detected the metastasis loci in the liver. All procedures of animal studies in the current study were approved by Ethics Committee 
of Guizhou Medical University and conformed to legal mandates and national guidelines for the care and maintenance of laboratory animals.

\section{Immunofluorescence staining}

Cells were fixed with $4 \%$ paraformaldehyde (Solarbio, Wuhan, China) for $15 \mathrm{~min}$. Then, the samples were cultured with anti-FUT11 (mouse source) , anti-N-cadherin(rabbit source), anti-E-cadherin(rabbit source), anti-HIF1a(rabbit source) and anti-PDK1 (rabbit source) primary antibody. After removing dissociative primary antibody by PBS, the samples were incubated with secondary antibodies anti-mouse conjugated FITC (Proteintech, Wuhan, China) and secondary antibodies anti-rabbit conjugated Cy3 (Proteintech, Wuhan, China). Nuclear were stained with DAPI (Boster, Wuhan, China). Finally, confocal microscopy or fluorescent microscope was used to collect the photo.

\section{Immunoprecipitation}

Cells were lysed in weak RIPA buffer (Proteintech, Wuhan, China) that included a 1\% PMSF (Boster, Wuhan, China). The liquid was centrifuged and the protein was collected. Then, the anti-FUT11 (1:50) antibody and IgG (1:50; Beyotime Biotechnology; Hangzhou, China) was added for 6 h, respectively. The, the $A / G$ agarose beads (Boster, Wuhan, China) was added for 3 h. After washing by PBS three times, isolated immunoprecipitates in beads were collected and analyzed using western blot.

\section{Luciferase assay}

After predicting binding site of HIF1a in the promoter of FUT11 using online database JASPAR(http://jaspar.binf.ku.dk/), dual luciferase reporter assay was performed to verify the bind. FUT11 promoter sequence with full-length and truncated were carried into the psi-basic luciferase reporter vector (Promega, USA). Finally, a total of $1 \times 10^{4}$ PANC-1 and AsPC-1 cells were plated into 24-well plate and cultured for overnight at $37^{\circ} \mathrm{C}$. Then, both of full-length and truncated FUT11 luciferase reporter vector and the si-HIF1a/si-NC were co-transfected into PC cells using lipidosome 2000 (Solarbio, Wuhan, China). After PC cells were cultured to normoxia or hypoxia, Finally, the luciferase activity of cells was determined after transfection at $24 \mathrm{~h}$.

\section{Statistical analysis}

SPSS software (version 21.0) was employed to perform statistical analysis. The difference between two groups was performed using paired t-test, while the difference among multiple groups were determined based on one-way analysis of variance. $P^{*}<0.05$ was used as cut-off to consider significant.

\section{Results}

FUT11 is a crucial hypoxia-related gene and up-regulated in PC tissues. 
To identify the key hypoxia-related genes, two gene data profile of PC cells (GSE67549 and GSE9350) under normoxia and hypoxia was analyzed. The results showed that there were 18 genes differently expressed of PC cells in hypoxia compared with that in normoxia (Fig. 1a). Among them, nine genes, ADM, C4orf3, ER01L, FUT11, BNIP3L, NDRG1, KCTD11, SLC2A1, P4HA1 were highly expressed in pancreatic cancer tissues compared to adjacent pancreatic tissues from TCGA and GTEx database (Fig. 1b). These 9 genes were considered as key hypoxia-related genes which involved in the progression of PC. Furthermore, we treated AsPC-1 and PANC-1 cells under normoxia and hypoxia, we found that these 9 genes were all increased under hypoxia compared with normoxia. Among them, a gene named FUT11 both increased the most significant ( $>3$ fold) in AsPC-1 and PANC-1 under hypoxia (Fig. 2a).

Then, we checked the expression level of FUT11 in human PC tissues and adjacent pancreatic tissues, the results showed that the expression of FUT11 was higher in pancreatic cancer tissues than that in adjacent pancreatic tissues (Fig. 2b-d). Similarly, We analyzed the correlation between FUT11 expression and the PC clinical pathology features,and showed that FUT11 expression was positive relatived with tumour size $(\mathrm{cm})$, lymph node metastasis, perineural invasion, blood vessel invasion and distant metastasis ability (Table 2). Further, Kaplan-Meier analysis show that the patients with higher FUT11 expression had poorer prognosis than those with lower FUT11 expression ( $P=0.014, \mathrm{HR}=1.884)$ (Fig. 2e).

\section{Suppression of FUT11 decreased PC cell proliferation and mobility in normoxia and hypoxia in vitro.}

Then, in order to uncover the effects of FUT11 on PC cells in normoxia and hypoxia, targeted FUT11 lentiviral was used to construct FUT11 knockdown cells. CCK-8 assay and EDU assay results showed that FUT11 knockdown obviously reduced the growth of AsPC-1 and PANC-1 cells under normoxia, as well as decreasing the stimulate impacts of hypoxia (Fig. 3a,b). Similarly, suppression of FUT11 obviously reduced the colony formation of AsPC-1 and PANC-1 cells in normoxia, as well as reducing the stimulate effects of hypoxia on colony formation (Fig. 3c). Transwell assay indicated that decreased the expression of FUT11 in AsPC-1 and PANC-1 cells obviously decreased the migration under normoxia and hypoxia (Fig. 3d). Western blot assay and immunofluorescence staining demonstrated that FUT11 knockdown decreased $\mathrm{N}$-cadherin expression and increased $\mathrm{E}$-cadherin expression in PC cells under normoxia and hypoxia (Fig. 3e,f). Take together, as a key hypoxia-related gene, FUT11 had potential to regulate the proliferation, mobility of PC cells in normoxia and hypoxia condition.

\section{Knockdown of FUT11 inhibits the PC cells proliferation and metastasis in vivo.}

The effects of FUT11 knockdown in vivo was also determined. We found that tumor tissues with FUT11 knockdown showed lower growth rate and tumor weight than negative control (all $P<0.05$, Fig. $4 a-c)$. We further assessed the expression of KI67 and PCNA in tumor tissues. Results indicated that KI67 and PCNA was obviously decreased in the tumor tissues with lower FUT11 expression (Fig. 4d). We then intrasplenically injected PANC-1 cells with FUT11 inhibition and negative control PANC-1 cells, and determined the effects of FUT11 on the hepatic metastasis of PANC-1 cells. Results showed that FUT11 knockdown significantly reduced the metastatic foci in the liver tissues (Fig. 4e-g). Furthermore, the 
FUT11 knockdown group had a markedly the longer survival time than the negative control group according to the survival analyses (Fig. 4h).

\section{FUT11 co-expressed with PDK1 and regulated the expression of PDK1 via maintaining the stability under hypoxia.}

In order to explore the molecular mechanism of FUT11 in PC, online database Genemania (https://genemania.org/) was used and predicted several proteins which binds with FUT11 directly (Fig. $5 a)$. Among them, PDK1 has been revealed as oncogene in pancreatic cancer. Therefore, we consider whether the effects of FUT11 on PC cells were associated with PDK1. Results of immunoprecipitation (IP) and immunofluorescence (IF) demonstrated that FUT11 was directly bound and co-localization with PDK1 (Fig. 5b-c). Similarly, the suppression of FUT11 prominent decreased the protein level of FUT11 under normoxia and hypoxia in AsPC-1 and PANC-1 cell (Fig. 5d). Previous studies showed that the members of FUT family can regulate the expression of related proteins via maintaining the stabilizing of their binding proteins[15]. Therefore, we consider that FUT11 may bind with PDK1 and protect it from degradation. To verify the guess, we used cycloheximide (CHX) to inhibit the synthesis of protein and detect the degradation rate of PDK1. The results are showen that the degradation rate of PDK1 was increased markedly in FUT11 knockdown group (Fig. 5e). Moreover, it is interesting that treatment with MG132 $(10 \mu \mathrm{M})$ restored the reduction of PDK1 induced by FUT11 suppression under hypoxia (Fig. $5 f)$. Furthermore, detecting the co-expression relationship between FUT11 and PDK1 in our PC samples, results indicated that there are co-expression relationship between them (Fig. $5 \mathrm{~g}$ ).

\section{PDK1 overexpression under hypoxia decreased the inhibitory effects of FUT11 knockdown}

To verify whether FUT11 promoted the proliferation of pancreatic cancer via decreasing the expression of PDK1, we overexpressed PDK1 in FUT11 knockdown PC cells. Then, we used CCK-8 and EDU method to detect the cell proliferation, and the results indicated that increased the expression of PDK1 significantly increased the proliferate rate of FUT11 low-expressed PC cells (Fig. 6a,b). Then we found that the colony formation ability of the cells co-transfected with targeting FUT11 lentivirus and PDK1 overexpression lentivirus under hypoxia was higher than that only transfected with targeting FUT11 lentivirus (Fig. 6c). Similarly, PDK1 overexpressed in FUT11 knockdown PC cells remarkably increased the mobility ability (Fig. 6d). Results of western blot showed that overexpressed PDK1 in FUT11 knockdown cells significantly rescued the protein level of N-cadherin and E-cadherin (Fig. 6e).

\section{FUT11 was determined as a target gene of HIF1a.}

Hypoxia-inducible factors including HIF1a were the most direct hypoxia response elements. To explore the regulated network of FUT11, we further determine whether FUT11 was directly regulated by HIF1a. After obtaining the motif of HIF1a in JASPAR database (Fig. 7a). we found that there is a hypoxiaresponsive element (HRE) in the promoter of FUT11 (Fig. 7b). The results indicated that compared with the control group, hypoxia treatment can significantly increased the luciferase activity in the cells transfected with the full-length FUT11 promoter vector, while the lack of HRE will damage the luciferase 
activity. Furthermore, inhibition of HIF1a reversed hypoxia-induced luciferase activity (Fig. 7c). Anti-HIF1a antibody was enrolled to perform ChIP assays in PANC-1 cells. Results indicated that the HRE in the FUT11 promoter was the major region mediating HIF1a-induced transcription (Fig. 7d). In addition, it is interesting that FUT11 was co-expressed with HIF1a in TCGA PC samples (Fig. 7e) and our additional PC samples (Fig. 7f).

\section{Restoration of FUT11 reversed the inhibitory effects of HIF1a on PC cells.}

To determine whether FUT11 was involved in the biology function of PC cells induced by HIF1a under hypoxia, we constructed negative control cells, FUT11 overexpressed cells, HIF1a knockdown cells and FUT11 overexpressed plus HIF1a knockdown cells, and treated them under hypoxia(Fig. 8a). CCK-8 and EDU assays results showed that HIF1a supression inhibited the proliferation of PC cells under hypoxia, while overexpression of FUT11 in HIF1a knockdown cells relieved the suppressive effects of HIF1a knockdown on growth (Fig. 8b-c). Similarly, the colony number of HIF1a inhibition group was obviously decreased. Overexpression of FUT11 in HIF1a knockdown cells relieved the inhibitory effects of HIF1a knockdown on colony formation ability under hypoxia (Fig. 8d). Furthermore, transwell assays demonstrated that HIF1a suppression remarkable decreased the mobility of PC cells in hypoxia, while overexpression of FUT11 in HIF1a knockdown cells reversed the inhibitory effects of HIF1a knockdown on cell migration and invasion (Fig. 8e).

\section{Discussion}

Even though the therapy for PC had been developed significantly, the prognosis of patients with PC was still poor[17]. Moreover, due to early metastasis, most PC patients lost the best time for therapy. Recently, increasing evidences showed that the distant metastasis in early stage of PC cells were drove by signal from tumor environment, including hypoxia[18]. Therefore, uncovering the mechanism of hypoxia regulated respond in PC cells was useful for the treatment of PC.

Previous studies had revealed that bioinformatics is a powerful instrument to identify genes associated the development of tumors, including PC[19]. Furthermore, online database GEO and TCGA storing thousands of gene database of tumor tissues provide enough analytical data. Therefore, in the current study, we tried to use the bioinformatics tool to identify novel hypoxia-related genes first. Through analyzing the gene expression profile, we found 18 genes were differently expressed between hypoxic PC samples and normoxic PC samples. Among these 18 genes, 9 of them including ADM, C4orf3, ERO1L, FUT11, BNIP3L, NDRG1, KCTD11, SLC2A1 and P4HA1 were highly expressed in PC tissues. Furthermore, FUT11 was increased the most significant in PC cells under hypoxia conditions, up-regulated in PC tissues and predicted poor prognosis for patients with PC. These were the first evidences provided from our current study that FUT11 may be a novel hypoxia-related gene.

The fucosyltransferase (FUT) family is the key enzymes in cell-surface antigen synthesis during various biological processes such as tumor resistance, proliferation and metastasis[20, 21]. Until now, total 13 members contained FUT1 to FUT11, protein O-fucosyltransferase 1 (POFUT1) and POFUT2 were 
identified. Various studies had demonstrated that some members of FUTs play as oncogenes in a series of cancer types. FUT8 was up-regulated in non-small cell lung cancer and promoted the process of epithelial-mesenchymal transition[22]. POFUT1 increased the activity of Notch1 signaling pathway and promoted the progression of colorectal cancer[23]. As shown in the previous studies, Inhibition of FUTs including FUT11 significantly decreased the expression and activity of ERK1/2 and p38 MAPK pathway, as well as the progression of human invasive ductal carcinoma[24]. FUT11 was highly expressed in gynecological cancer, and higher expression of FUT11 in patient predicted poor outcome[25]. However, the effects of the FUT11 on proliferation and metastasis have not yet been clearly defined in human PC cells. Consistent with previous study, using CCK-8 assay, colony formation assay and wound healing assay, we found that FUT11 inhibition significantly decreased proliferation and migration of PC cells in both hypoxic and normoxic environment. Via performing xenograft tumor model and in vivo metastasis model, we found that FUT11 inhibition decreased the PC cells proliferation and metastasis in vivo. These were first experiment evidences that FUT11 linked to hypoxia, since it had potential to regulate PC cells proliferation and migration under normoxia and hypoxia.

PDK1 has emerged as an important oncogene in many cancers including PC [26]. Lucero-Acuna A et al has been reported that the expression of PDK1 is up-regulated in human PC and promotes cancer cell growth and mobility[27]. Xia S et a/ knockdown of PDK1, forces cells containing activated p21(Ras) to undergo apoptosis in PC cells[28]. However, the mechanisms regulating PDK1 expression during tumor progression is not clear at present. In the current study, through bioinformatics analysis, PDK1 was identified as one of potential downstream genes of FUT11, which co-expressed with FUT11. Further, using immunoprecipitation and western blot, we found that FUT11 directly bind with PDK1 and regulated its expression in normoxia and hypoxia. As the knowledge obtained from previous studies, FUTs can bind with a series of proteins and maintain their stability via blocking the bind site of protease[15]. Therefore, we then determine whether FUT11 regulated PDK1 via maintaining its stability. Consistent with our speculation, knockdown of FUT11 under hypoxia increased the degradation rate of PDK1. Furthermore, overexpression of PDK1 in PC under hypoxia relieved the inhibitory impacts of FUT11 knockdown on cell proliferation and migration.

The relationship between hypoxia-inducible factor, hypoxia microenvironment, hypoxia related to genes and the development of PC were widely reported in previous study. For example, PAFAH1B2 regulated by HIF1a under hypoxia promoted the growth and mobility of PC cells[29]. MTA1 was regulated via HIFa/VEGF axis and promoted the development of PC[30]. Similarly, Overexpression of hydroxyproline via EGLN/HIF1A is associated with distant metastasis in PC[31]. Similarly, our early study also demonstrated that YEATS2 directly targets HIF1 a which promotes PC cellS proliferation and mobility[32]. In the present study, we provided the first evidences that FUT11 was a novel target gene of HIF1a, which involved in the biology function mediating by HIF1a under hypoxia.

In conclusion, our present study demonstrated that FUT11 was a new hypoxia related gene, which high expresssion in pancreatic cancer tissues and related to poor prognosis in pancreatic cancer patients. 
FUT11 was regulated via HIF1a and promoted PC cells proliferation and migration via maintaining the stability of PDK1. FUT11 may be an effective target for overcoming the hypoxia respond of PC.

\section{Conclusions}

The fucosyltransferase 11 (FUT11) was a direct target gene of hypoxia-inducible factor 1a (HIF1a), and it was up-regulated in pancreatic cancer cells under hypoxia, which can promote the proliferation and metastasis of pancreatic cancer cells via maintaining the stability of transcription factor 1 (PDK1) under hypoxia.

\section{Abbreviations}

TCGA: The Cancer Genome Atlas

GTEx : The Genotype-Tissue Expression

PC: pancreatic cancer

GEO: Gene Expression Omnibus

FUT: fucosyltransferase

PDK1: pyruvate dehydrogenase kinase 1

HIF1a: Hypoxia-inducible factor $1 a$

EDU: 5-ethynyl-2'-deoxyuridine

IF: immunofluorescence

IP: immunoprecipitation

qRT-PCR: Real-time quantitative reverse transcription PCR

CCK-8: Cell counting kit

\section{Declarations}

\section{Ethics approval and consent to participate}

This study was reviewed and approved by the Medical Ethics Committee of Guizhou Medical University, Wuhan, China. All animal studies were approved by the Institutional Animal Care and Use Committee of Committee of Guizhou Medical University. 
All authors have agreed to publish this manuscript.

\section{Availability of data and material}

The datasets used and/or analyzed during the current study are available from the corresponding author on reasonable request.

\section{Competing interests}

The authors declare that they have no competing interests.

\section{Funding}

This work was supported by the Non-profit central research Institute fund of Chinese academy of medical sciences 2019PT320003, grant from the Department of talents of Guizhou Province, grants from Guizhou Science and Technology Cooperation Platform (2017-5724, 2018-5706), and National Natural Science Foundation of China (82060308), and National Natural Science Foundation of China (82060491).

\section{Authors' contributions}

WP, ZR , ZJ and SL contributed to the experiment design, and data analysis. RS, ZW, HW, WP and SL contributed to the experiment implementation, YJ, XG, HC, PW, XY and SL contributed to manuscript draft and data analysis. All authors read and approved the final manuscript.

\section{Acknowledgements}

We acknowledge the generous support of the department of hepatobiliary surgery, Guizhou Medical University, Guiyang, China.

\section{References}

1. Lee KG, Roy V, Laszlo M, Atkins KM, Lin KJ, Tomassian S, Hendifar AE: Symptom Management in Pancreatic Cancer. Curr Treat Options Oncol 2021, 22:8.

2. Kishimoto S, Brender JR, Chandramouli GVR, Saida Y, Yamamoto K, Mitchell JB, Krishna MC: Hypoxia-Activated Prodrug Evofosfamide Treatment in Pancreatic Ductal Adenocarcinoma Xenografts Alters the Tumor Redox Status to Potentiate Radiotherapy. Antioxid Redox Signal 2020.

3. Hu M, Kang G, Cheng X, Wang J, Li R, Bai Z, Yang D, Huang H: In vitro affinity maturation to improve the efficacy of a hypoxia-inducible factor 1alpha singledomain intrabody. Biochem Biophys Res Commun 2020, 529:936-942.

4. Wozniak M, Pastuch-Gawolek G, Makuch S, Wisniewski J, Ziolkowski P, Szeja W, Krawczyk M, Agrawal S: Overcoming Hypoxia-Induced Chemoresistance in Cancer Using a Novel Glycoconjugate of Methotrexate. Pharmaceuticals (Basel) 2020, 14. 
5. Zhang Q, Han Z, Zhu Y, Chen J, Li W: Role of hypoxia inducible factor-1 in cancer stem cells (Review). Mol Med Rep 2021, 23.

6. Guo FJ, Shao YP, Wang YP, Jin YM, Liu SS, Wang QY: MIR-92 stimulates VEGF by inhibiting von Hippel-Lindau gene product in epithelial ovarian cancer. J Biol Regul Homeost Agents 2017, 31:615624.

7. Ebright RY, Zachariah MA, Micalizzi DS, Wittner BS, Niederhoffer KL, Nieman LT, Chirn B, Wiley DF, Wesley B, Shaw B, et al: HIF1A signaling selectively supports proliferation of breast cancer in the brain. Nat Commun 2020, 11:6311.

8. Silva P, Slevin NJ, Sloan P, Valentine H, Cresswell J, Ryder D, Price P, Homer JJ, West CM: Prognostic significance of tumor hypoxia inducible factor-1alpha expression for outcome after radiotherapy in oropharyngeal cancer. Int J Radiat Oncol Biol Phys 2008, 72:1551-1559.

9. Kutkowska J, Strzadala L, Rapak A: Hypoxia increases the apoptotic response to betulinic acid and betulin in human non-small cell lung cancer cells. Chem Biol Interact 2021, 333:109320.

10. Cui XG, Han ZT, He SH, Wu XD, Chen TR, Shao CH, Chen DL, Su N, Chen YM, Wang T, et al: HIF1/2alpha mediates hypoxia-induced LDHA expression in human pancreatic cancer cells. Oncotarget 2017, 8:24840-24852.

11. Fan LF, Diao LM, Chen DJ, Liu MQ, Zhu LQ, Li HG, Tang ZJ, Xia D, Liu X, Chen HL: [Expression of HIF1 alpha and its relationship to apoptosis and proliferation in lung cancer]. Ai Zheng 2002, 21:254258.

12. Wang Z, Li Q, Xia L, Li X, Sun C, Wang Q, Cai X, Yang G: Borneol promotes apoptosis of Human Glioma Cells through regulating HIF-1a expression via mTORC1/elF4E pathway. J Cancer2020, 11:4810-4822.

13. Hwang H, Jeong HK, Lee HK, Park GW, Lee JY, Lee SY, Kang YM, An HJ, Kang JG, Ko JH, et al: Machine Learning Classifies Core and Outer Fucosylation of N-Glycoproteins Using Mass Spectrometry. Sci Rep 2020, 10:318.

14. Liang L, Gao C, Li Y, Sun M, Xu J, Li H, Jia L, Zhao Y: miR-125a-3p/FUT5-FUT6 axis mediates colorectal cancer cell proliferation, migration, invasion and pathological angiogenesis via PI3K-Akt pathway. Cell Death Dis 2017, 8:e2968.

15. Kumar S, Das S, Rachagani S, Kaur S, Joshi S, Johansson SL, Ponnusamy MP, Jain M, Batra SK: NCOA3-mediated upregulation of mucin expression via transcriptional and post-translational changes during the development of pancreatic cancer. Oncogene 2015, 34:4879-4889.

16. Lin G, Zhao R, Wang Y, Han J, Gu Y, Pan Y, Ren C, Ren S, Xu C: Dynamic analysis of N-glycomic and transcriptomic changes in the development of ovarian cancer cell line A2780 to its three cisplatinresistant variants. Ann Trans/ Med 2020, 8:289.

17. Kurihara K, Hanada K, Shimizu A: Endoscopic Ultrasonography Diagnosis of Early Pancreatic Cancer. Diagnostics (Basel) 2020, 10.

18. Bhandari V, Hoey C, Liu LY, Lalonde E, Ray J, Livingstone J, Lesurf R, Shiah YJ, Vujcic T, Huang X, et al: Molecular landmarks of tumor hypoxia across cancer types. Nat Genet 2019, 51:308-318. 
19. Lv K, Yang J, Sun J, Guan J: Identification of key candidate genes for pancreatic cancer by bioinformatics analysis. Exp Ther Med 2019, 18:451-458.

20. Gan CZ, Li G, Luo QS, Li HM: miR-339-5p downregulation contributes to Taxol resistance in small-cell lung cancer by targeting alpha1,2-fucosyltransferase 1. IUBMB Life 2017, 69:841-849.

21. Wang S, Zhang $X$, Yang $C$, Xu S: MicroRNA-198-5p inhibits the migration and invasion of non-small lung cancer cells by targeting fucosyltransferase 8. Clin Exp Pharmacol Physio/ 2019, 46:955-967.

22. Li F, Zhao S, Cui Y, Guo T, Qiang J, Xie Q, Yu W, Guo W, Deng W, Gu C, Wu T: alpha1,6Fucosyltransferase (FUT8) regulates the cancer-promoting capacity of cancer-associated fibroblasts (CAFs) by modifying EGFR core fucosylation (CF) in non-small cell lung cancer (NSCLC). Am J Cancer Res 2020, 10:816-837.

23. Du Y, Li D, Li N, Su C, Yang C, Lin C, Chen M, Wu R, Li X, Hu G: POFUT1 promotes colorectal cancer development through the activation of Notch1 signaling. Cell Death Dis 2018, 9:995.

24. Carrascal MA, Silva M, Ramalho JS, Pen C, Martins M, Pascoal C, Amaral C, Serrano I, Oliveira MJ, Sackstein R, Videira PA: Inhibition of fucosylation in human invasive ductal carcinoma reduces Eselectin ligand expression, cell proliferation, and ERK1/2 and p38 MAPK activation. Mol Oncol 2018, 12:579-593.

25. Zhang $X$, Wang $Y$ : Identification of hub genes and key pathways associated with the progression of gynecological cancer. Oncol Lett 2019, 18:6516-6524.

26. Wang C, Liu H, Qiu Q, Zhang Z, Gu Y, He Z: TCRP1 promotes NIH/3T3 cell transformation by overactivating PDK1 and AKT1. Oncogenesis 2017, 6:e323.

27. Lucero-Acuna A, Jeffery JJ, Abril ER, Nagle RB, Guzman R, Pagel MD, Meuillet EJ: Nanoparticle delivery of an AKT/PDK1 inhibitor improves the therapeutic effect in pancreatic cancer. Int $J$ Nanomedicine 2014, 9:5653-5665.

28. Xia S, Chen Z, Forman LW, Faller DV: PKCdelta survival signaling in cells containing an activated P21Ras protein requires PDK1. Cell Signal 2009, 21:502-508.

29. Ma C, Guo Y, Zhang Y, Duo A, Jia Y, Liu C, Li B: PAFAH1B2 is a HIF1a target gene and promotes metastasis in pancreatic cancer. Biochem Biophys Res Commun 2018, 501:654-660.

30. Sun X, Zhang Y, Li B, Yang H: MTA1 promotes the invasion and migration of pancreatic cancer cells potentially through the HIF-alpha/VEGF pathway. J Recept Signal Transduct Res 2018, 38:352-358.

31. Chiba N, Sunamura M, Nakagawa M, Koganezawa I, Yokozuka K, Kobayashi T, Hikita K, Ozawa Y, Okihara M, Sano T, et al: Overexpression of hydroxyproline via EGLN/HIF1A is associated with distant metastasis in pancreatic cancer. Am J Cancer Res 2020, 10:2570-2581.

32. Zeng Z, Lei S, He Z, Chen T, Jiang J: YEATS2 is a target of HIF1alpha and promotes pancreatic cancer cell proliferation and migration. J Cell Physio/ 2021, 236:2087-2098.

\section{Tables}


Due to technical limitations, table 1 and table 2 is only available as a download in the Supplemental Files section.

\section{Figures}

A

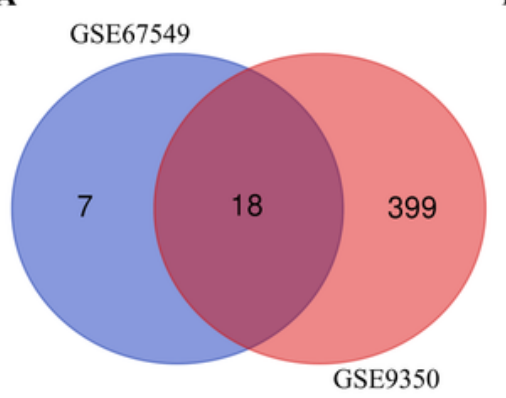

B

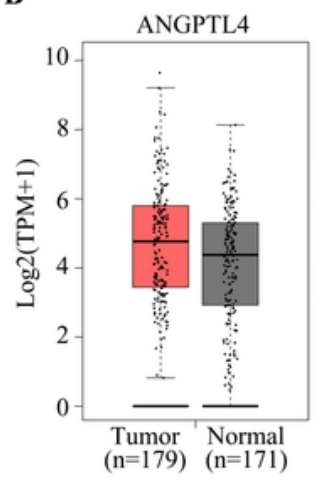

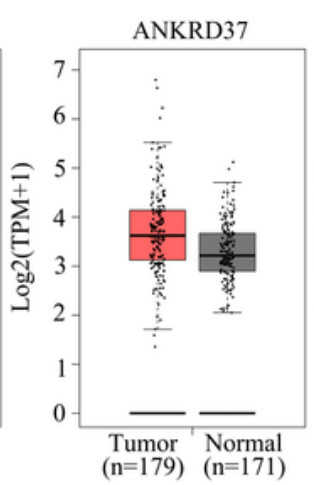

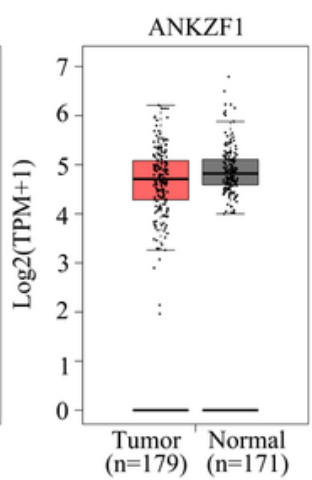

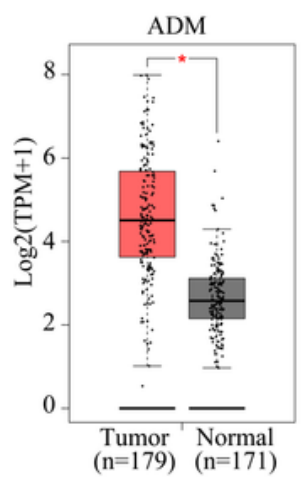
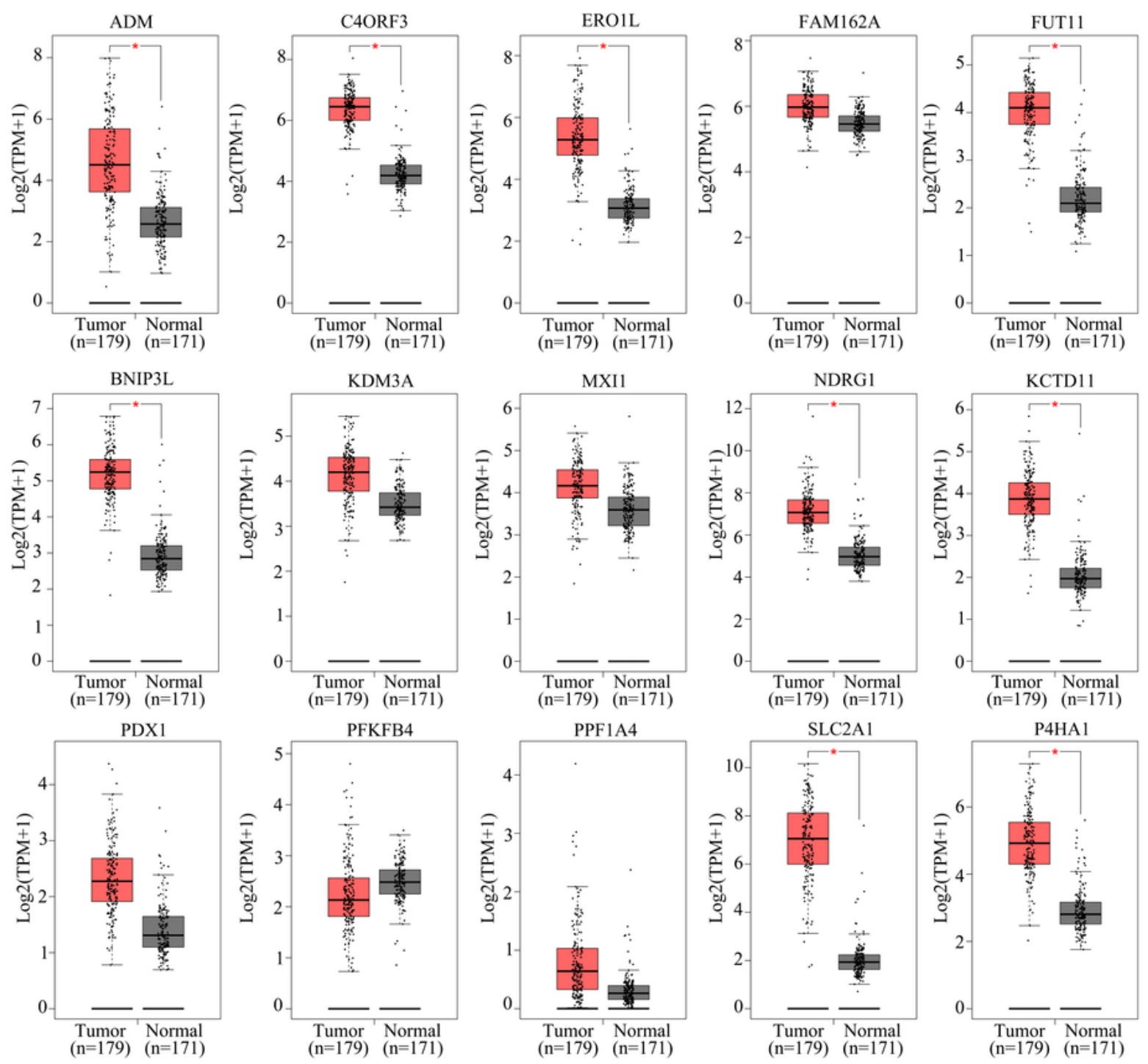

Figure 1 
Identification of key hypoxia-related genes in PC. a Intersection analysis of differently expressed genes in PC samples under hypoxia according to the gene expression profiles GSE67549 and GSE9350. b ANGPTL4, ANKRD37, ANKZF1, ADM, C4ORF3, ER01L, FAM162A, FUT11, BNIP3L, KDM3A, MXI1, NDRG1, KCTD11, PDX1, PFKFB1, PPF1A4, SLC2A1 and P4HA1 was highly expressed in PC tissues compared with non-tumor tissues analyzed by GEPIA online tool.

A

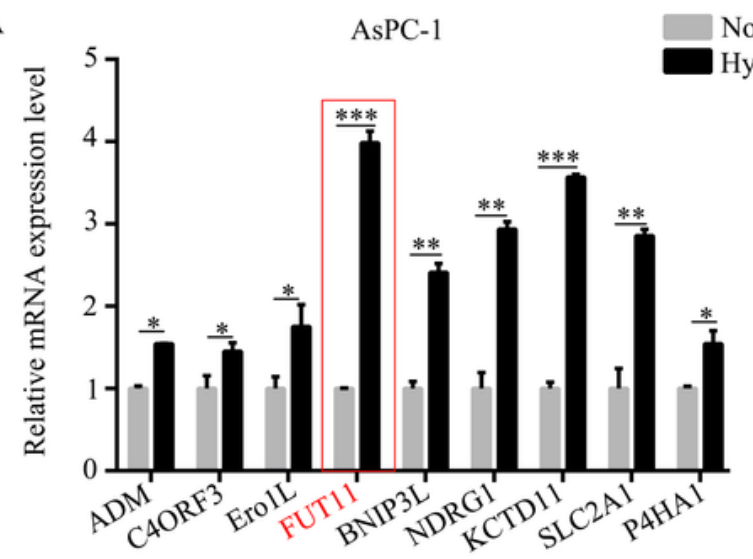

B

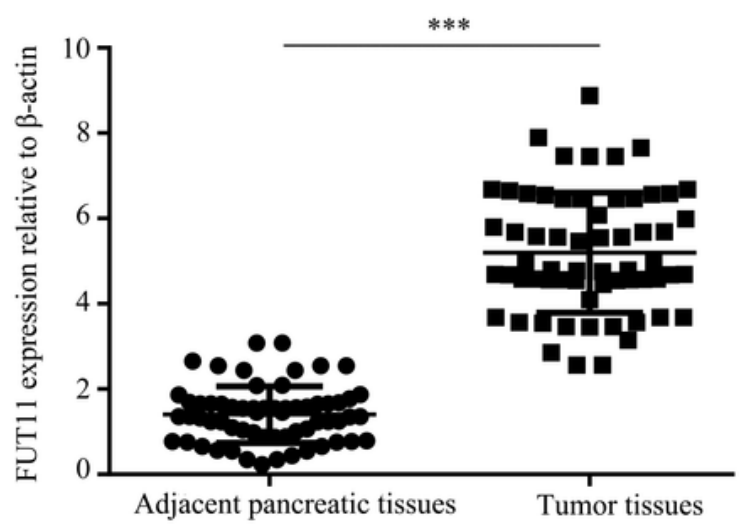

D

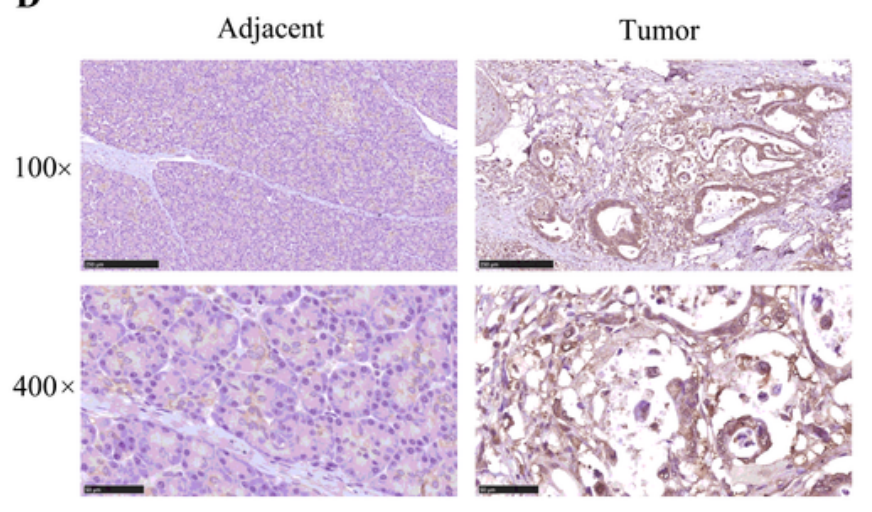

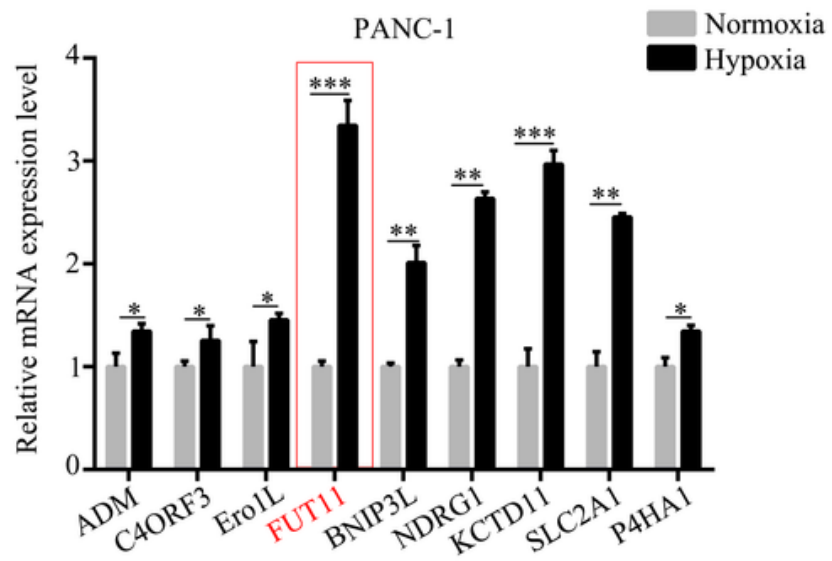

C

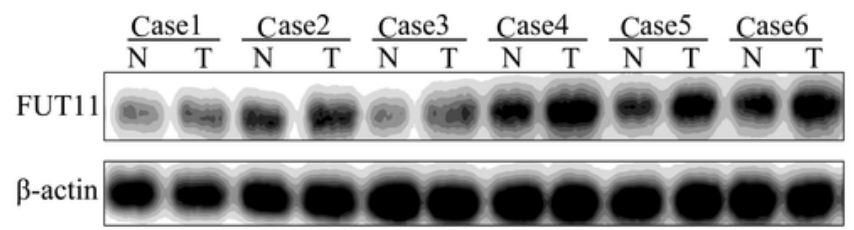

$\mathbf{E}$

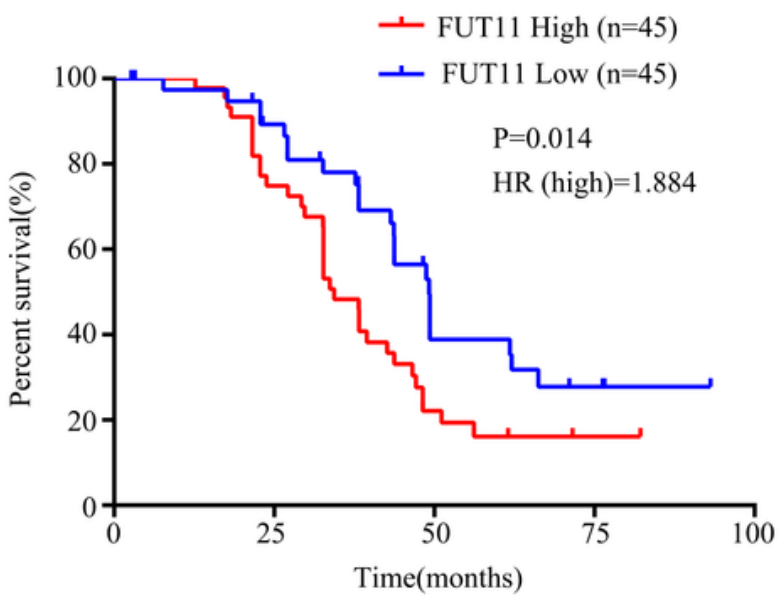

Figure 2 
FUT11 was up-regulated in PC and predicted poor outcome. a qRT-PCR was used to detect the expression of ADM, C4ORF3, ER01L, FUT11 BNIP3L, KDM3A, MXI1, NDRG1 and KCTD11 in AsPC-1 and PANC-1 cells under normoxia and hypoxia. b qRT-PCR analysis of mRNA level of FUT11 in 62 pairs of matched PC tissue and adjacent normal tissues. c western blot analysis of protein level of FUT11 in PC tissue and adjacent normal tissues. $\mathrm{d}$ IHC analysis of the expression level of FUT11 in pancreatic cancer and normal pancreatic tissue. e 90 patients with PC were averagely divided into two groups based on the expression of FUT11. Kaplan survival curve showed the overall survival rate of high FUT11(red) and low FUT11(blue) expression group. * $\mathrm{P}<0.05 ;$; $\mathrm{P}<0.01$; $* \star \star \mathrm{P}<0.001$. 

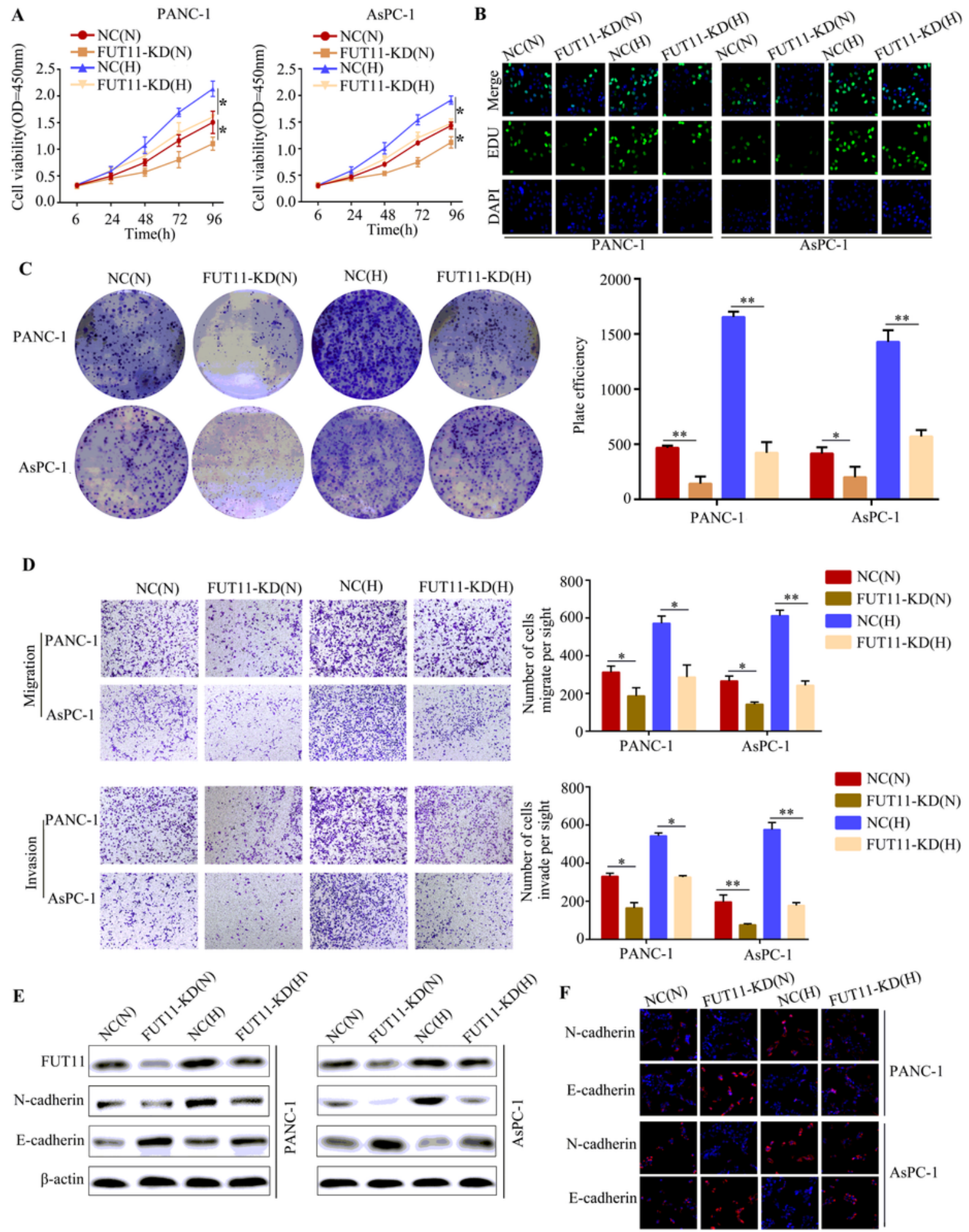

\section{Figure 3}

FUT11 regulated the proliferation, invasion and migration of PC cells under normoxia and hypoxia in vitro. Targeted FUT11 lentiviral and negative lentiviral were used to construct FUT11 knockdown cells and negative control cells, which were cultured either in normoxia and hypoxia. a The effect of FUT11 on PC cell proliferation was detected by CCK-8 method. b The effect of FUT11 on PC cell proliferation viability was detected by EDU assay. c The effect of FUT11 on PC cell colon formation viability was detected by 
colon formation assay. $d$ The effect of FUT11 on PC cell invasion and migration viability was detected by Transwell assays. e Western blotting was used to detect the protein level of N-cadherin and E-cadherin of each group. $\mathrm{f}$ Immunofluorescent staining assay to detect the expression of $\mathrm{N}$-cadherin and E-cadherin of each group. ${ }^{*} \mathrm{P}<0.05 ; * * \mathrm{P}<0.01$.

A

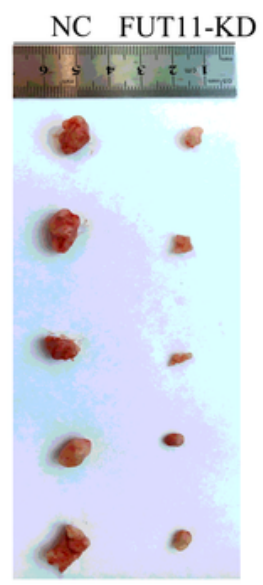

D
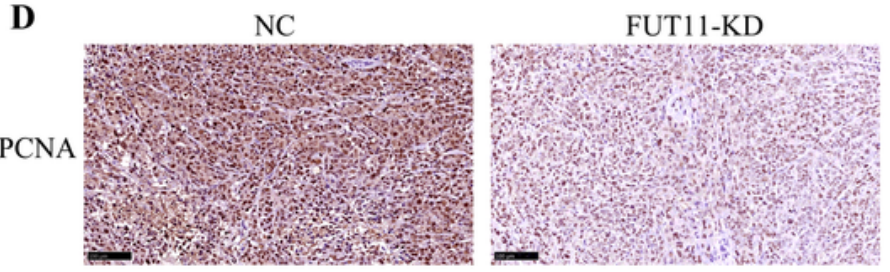

Ki-67
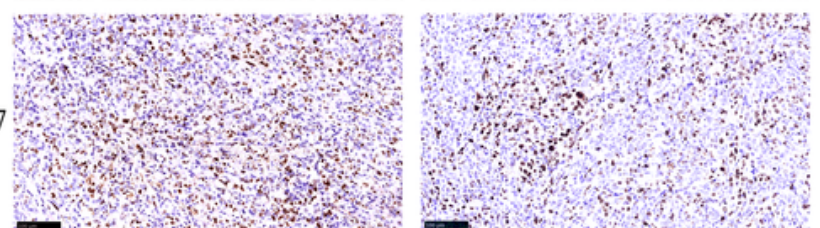

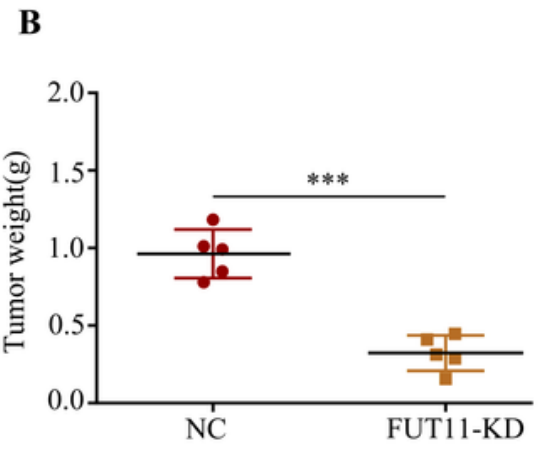

C

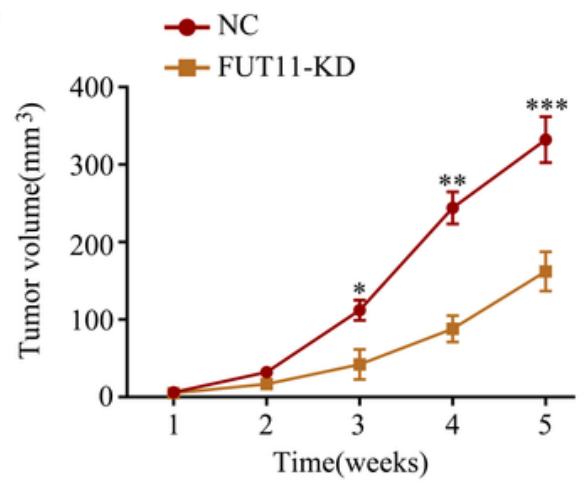

$\mathbf{E}$
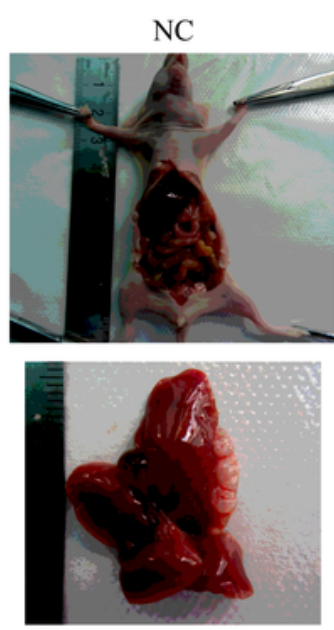

FUT11-KD
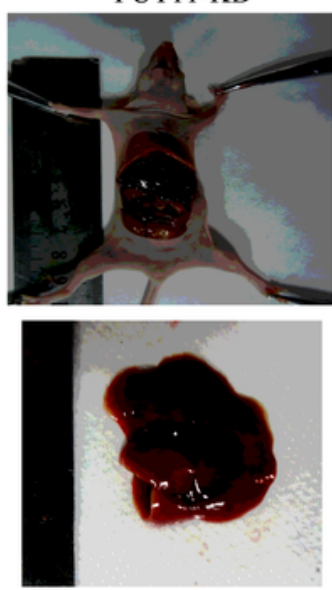

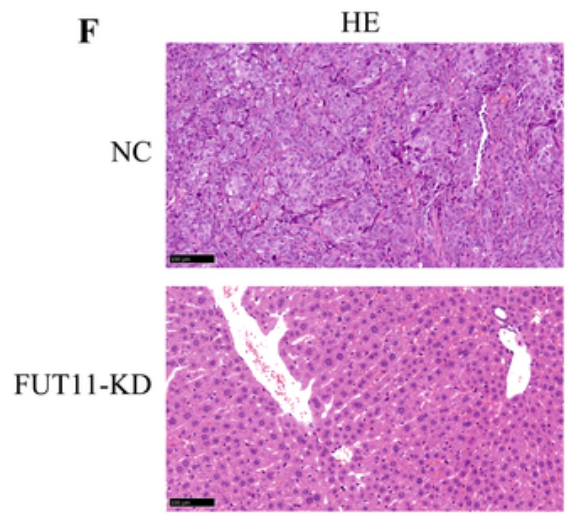

G

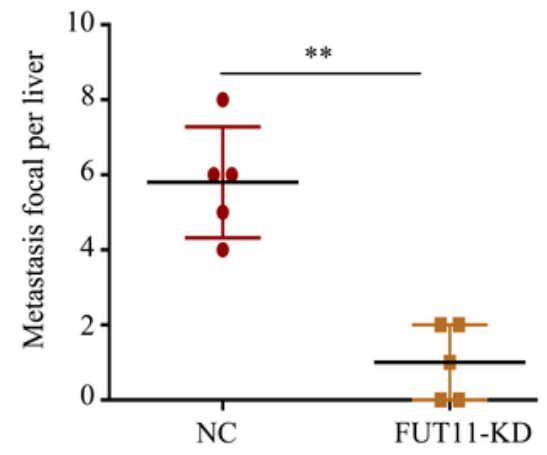

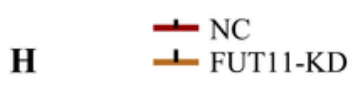

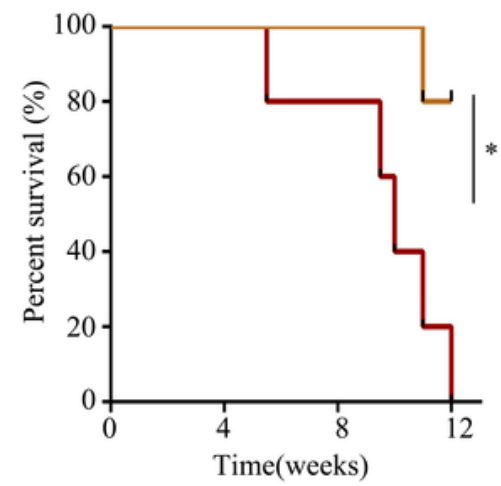

Figure 4 
Inhibition of FUT11 suppressed the proliferation and metastatic of PANC-1 cells in vivo. a Typical image of tumor tissues in negative control group and FUT11 knockdown group. b The mean weight of tumor tissues with FUT11 knockdown and negative control. c The proliferate rate of tumor tissues with FUT11 knockdown and negative control. d Typical IHC staining images showing Ki-67 and PCNA expression in transplanted tumors under different experimental conditions. e Image of liver metastasis model $(n=5)$. $f$ Typical IHC staining of HE images showing metastasis loci in liver in indicated groups. $\mathrm{g}$ Statistical analysis of the average numbers of visible liver metastases foci. $\mathrm{h}$ Kaplan-Meier survival curves for each experimental group, $(n=5) .{ }^{*} P<0.05 ; * \star P<0.01$. 
A

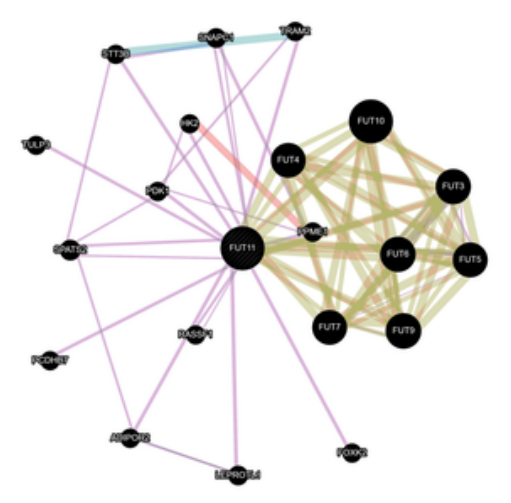

B

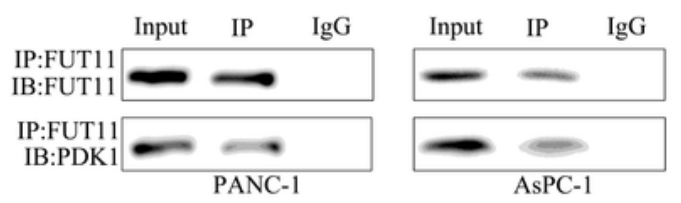

C

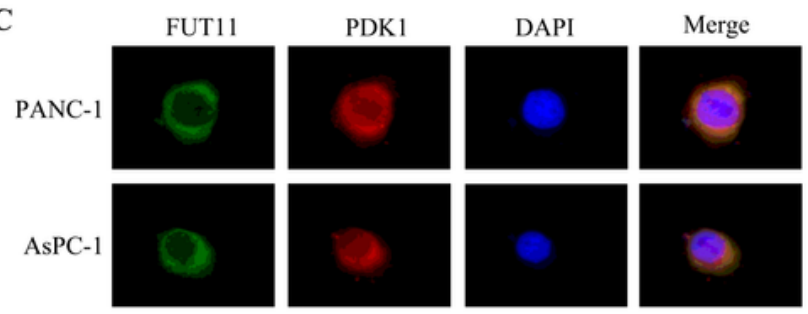

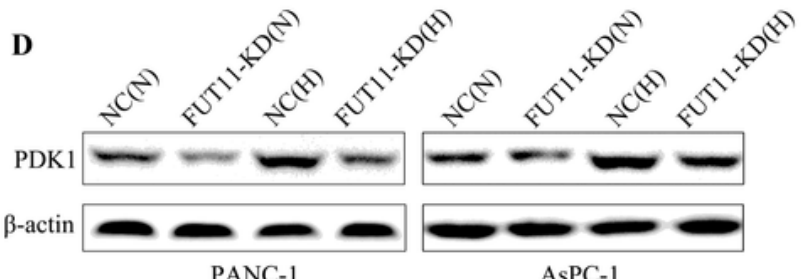

$\mathbf{E}$

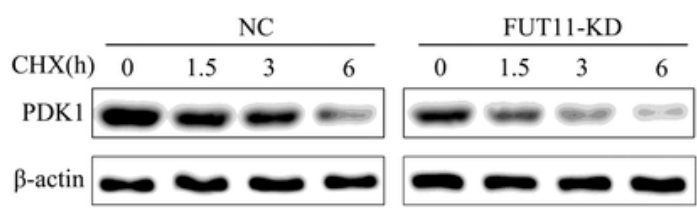

PANC-1

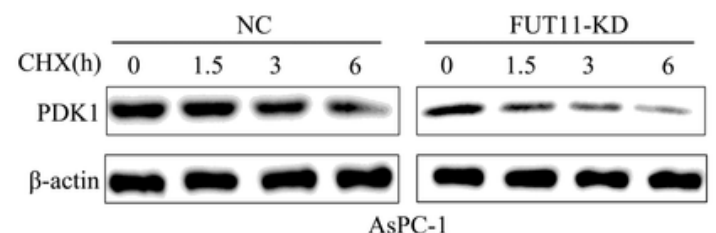

F

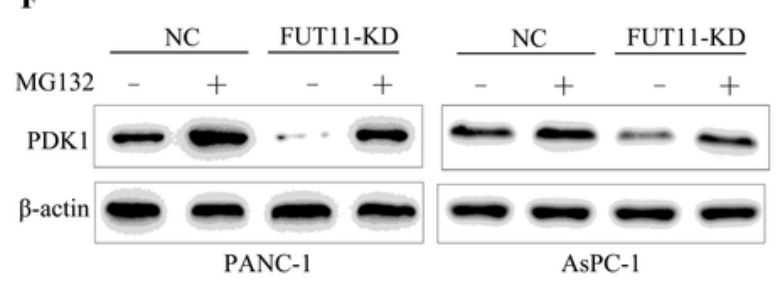

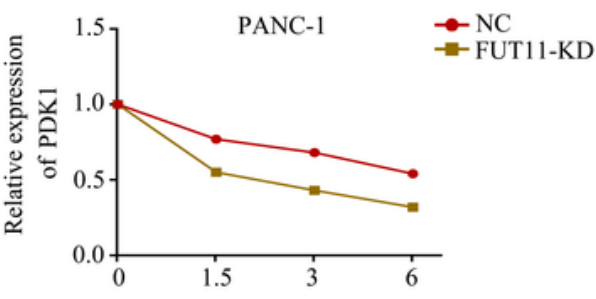
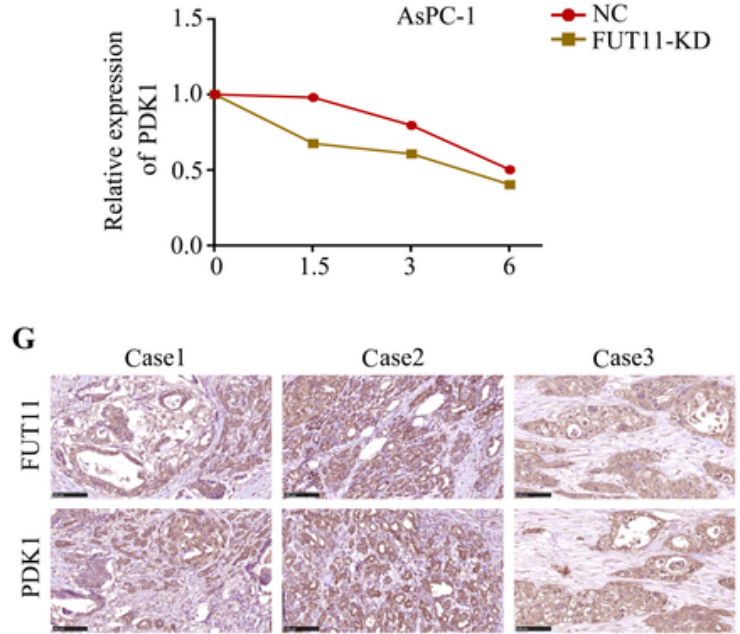

\section{Figure 5}

FUT11 bind with PDK1 and regulated its expression. a The predicted potential binding of FUT11 with PDK1 via online database Genemania. $b$ Immunoprecipitation was used to analyze the bind between FUT11 and PDK1. c Immunofluorescence showed that FUT11 co-localized with PDK1 in PC cells. d Western blot was used to detect the expression of FUT11 in sh-scramble and FUT11 knockdown PC cells under normoxia and hypoxia. e $\mathrm{CHX}$ was used to inhibit the protein synthesis, and the degradation rate of 
PDK1 in sh-scramble and FUT11 knockdown cells under hypoxia was detected using western blot. $f$ Western blot was used to the expression of PDK1 in sh-scramble and FUT11 cells treatment with MG132 under hypoxia. g Typical immunohistochemical figures showed the co-expression relationship between FUT11 and PDK1.
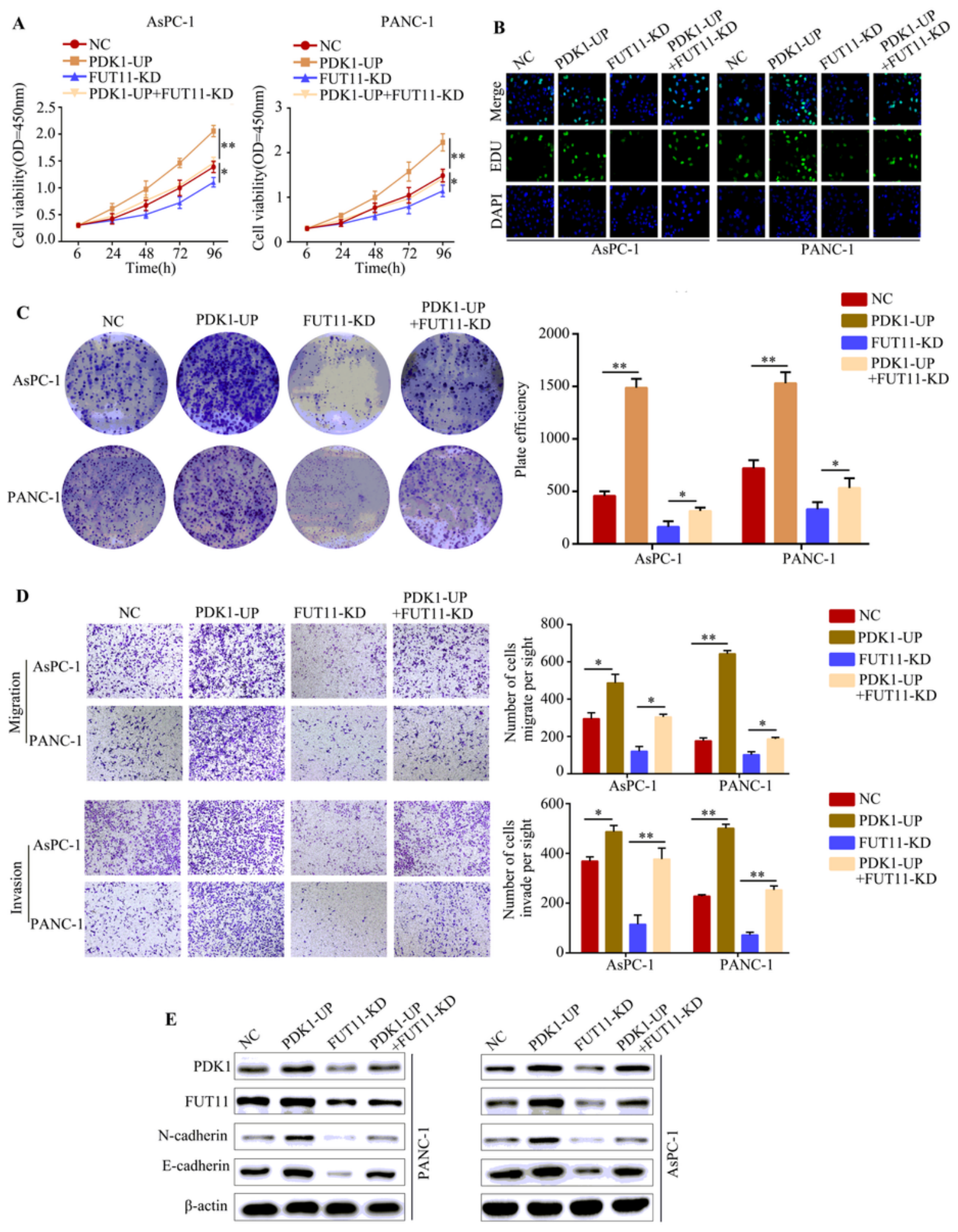

Figure 6 
Overexpression of PDK1 reversed the inhibitory effects of FUT11 inhibition on PC cell proliferation, invasion and migration. According to the treatment factors, cells were divided into four groups: negative control group (NC), PDK1 overexpressed group (PDK1-UP), FUT11 knockdown group (FUT11-KD) and PDK1 overexpressed plus FUT11 knockdown group (PDK1-UP + FUT11-KD). All cells were cultured in hypoxia. a CCK-8 was used to detect the proliferation ability of each group. b EDU assay was used to detect the proliferation of each group. c Colony formation was used to detect the colony formation ability of each group. $d$ Transwell assay was used to detect the migration and invasion ability of each group. $e$ Western blot was used to detect the expression of FUT11, PDK1, N-cadherin and E-cadherin in each group. * $\mathrm{P}<0.05$; $* * \mathrm{P}<0.01$; *** $\mathrm{P}<0.001$.

A

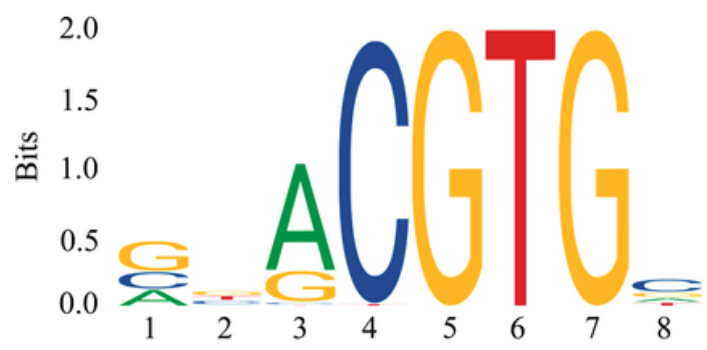

B

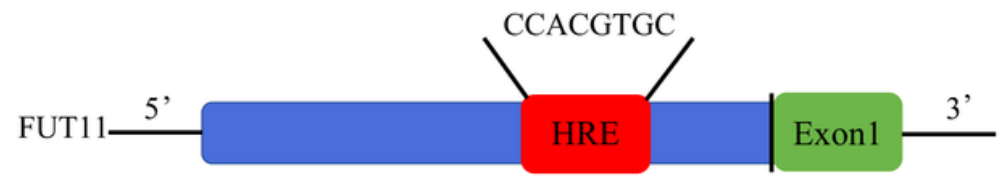

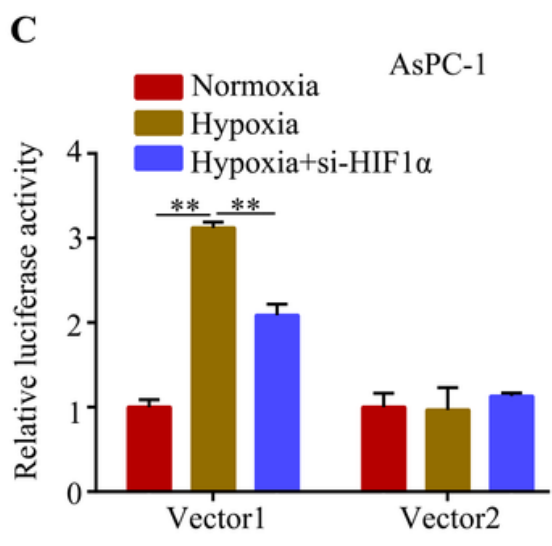

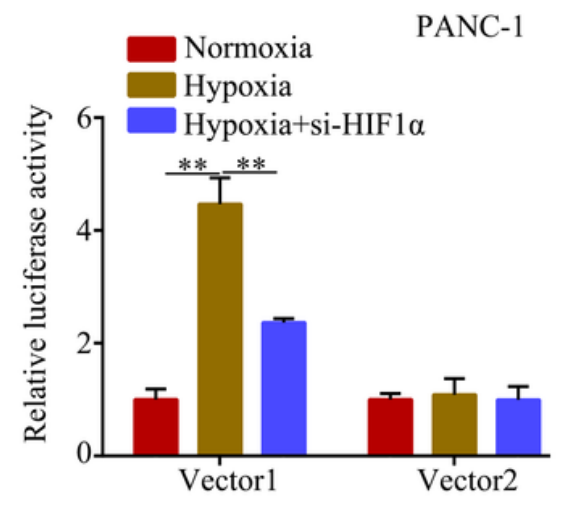

D

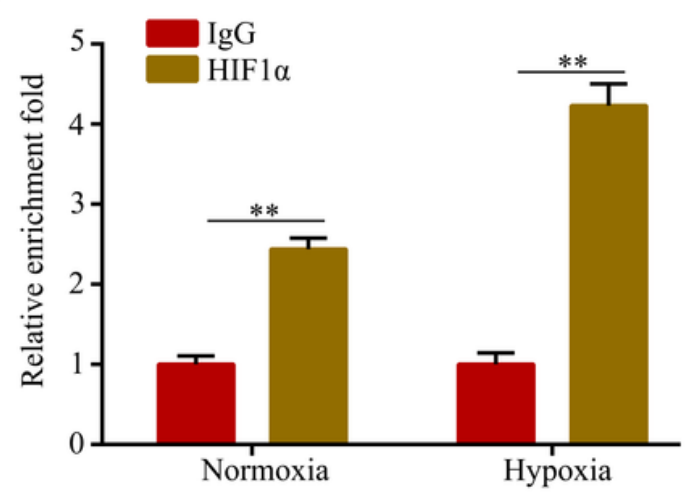

E

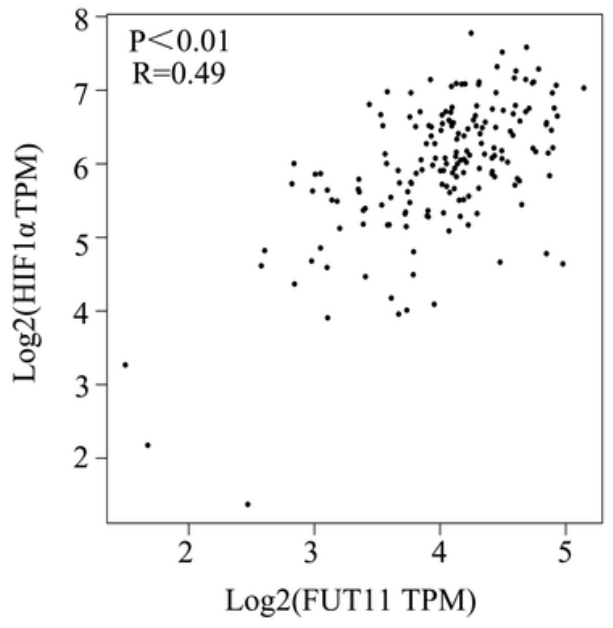

F

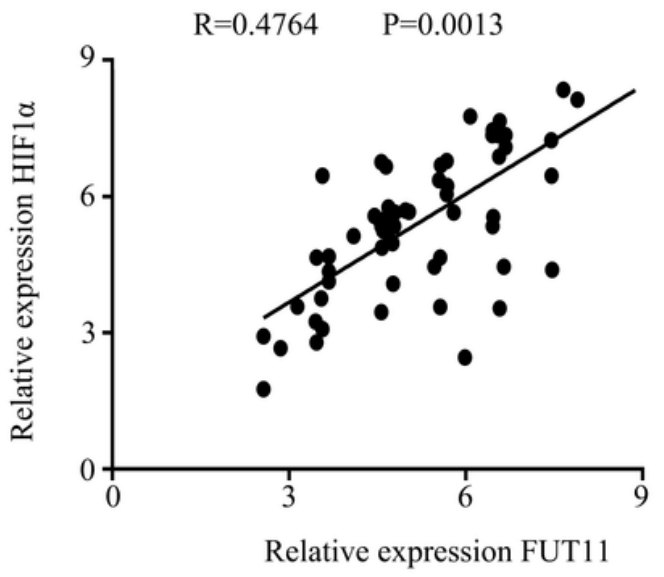




\section{Figure 7}

HIF1 a regulates the expression of FUT11 by binding to the HRE of the FUT11 promoter. a The motif of HIF1 a was showed. b The hypoxia response element in the promoter of FUT11 was showed. c PANC-1 and AsPC-1 cells were transfected with either a full-length or truncated FUT11 promoter-pGL3 reporter vector and further cultured under hypoxia, either with or without si-HIF1a. After 48 hours, luciferase activity was measured using the dual-luciferase reporter assay system. d ChIP assays with anti-HIF1a antibody verifying the binding between HIF1 $\mathrm{a}$ and hypoxia response element of the FUT11 promoter under normoxia and hypoxia. e Co-expression relationship between FUT11 and HIF1a based on the data from PC tissues via online database GEPIA. $f$ Co-expression relationship between FUT11 and HIF1a based on the data from our additional PC tissues $(n=62)$. ${ }^{*}<0.05, * \star P<0.01$. 

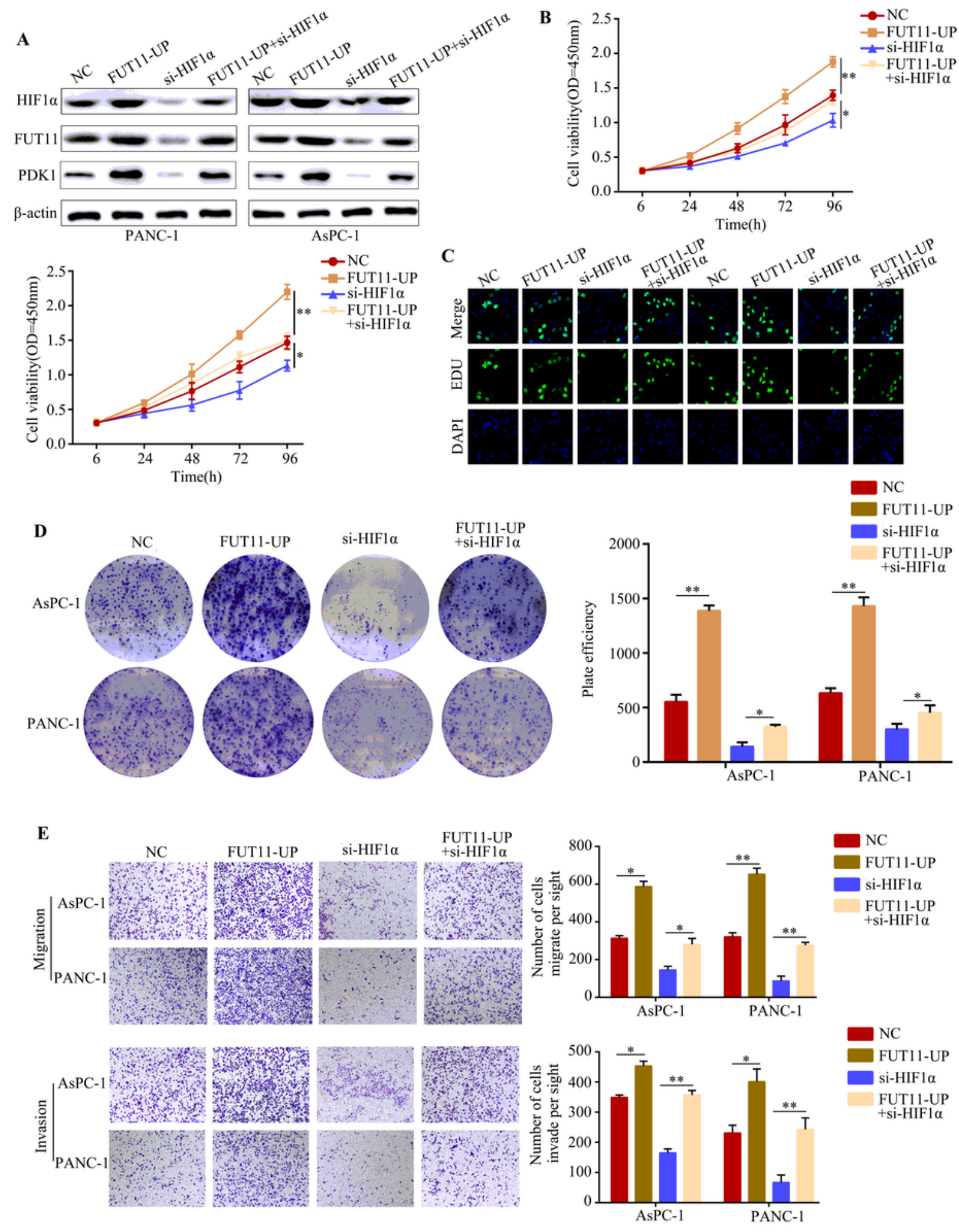

\section{Figure 8}

Overexpression of FUT11 reversed the inhibitory effects of HIF1a knockdown on PC cell proliferation, invasion and migration under hypoxia. PANC-1 and AsPC-1 cells were divided into four groups as follow: negative control (NC); FUT11 overexpression (FUT11-UP); HIF1a inhibition (si-HIF1a); HIF1a inhibition plus FUT11 overexpression (si-HIF1a+FUT11-UP). All group cells were cultured in hypoxia. a Western blot was used to detect the expression of HIF1a and FUT11 in each group cells. b CCK-8 methods were used 
to detect the proliferation ability in each group cells. c EDU assay was used to detect the proliferation ability in each group cells. d Colony formation assays were used to detect the colony formation ability in each group cells. e Transwell assays were used to detect the invasion and migration ability in each group cells. The data are shown as means \pm S.D. of three independent assays. ${ }^{*} P<0.05,{ }^{\star} * P<0.01$.

\section{Supplementary Files}

This is a list of supplementary files associated with this preprint. Click to download.

- Table1.xlsx

- Table2.xlsx 